\begin{tabular}{|l|l|l||}
\hline \multicolumn{2}{|c|}{ PublisherInfo } \\
\hline \hline PublisherName & $:$ & BioMed Central \\
\hline \hline PublisherLocation & $:$ & London \\
\hline \hline PublisherImprintName & $:$ & BioMed Central \\
\hline \hline
\end{tabular}

\title{
CD44 and Fas
}

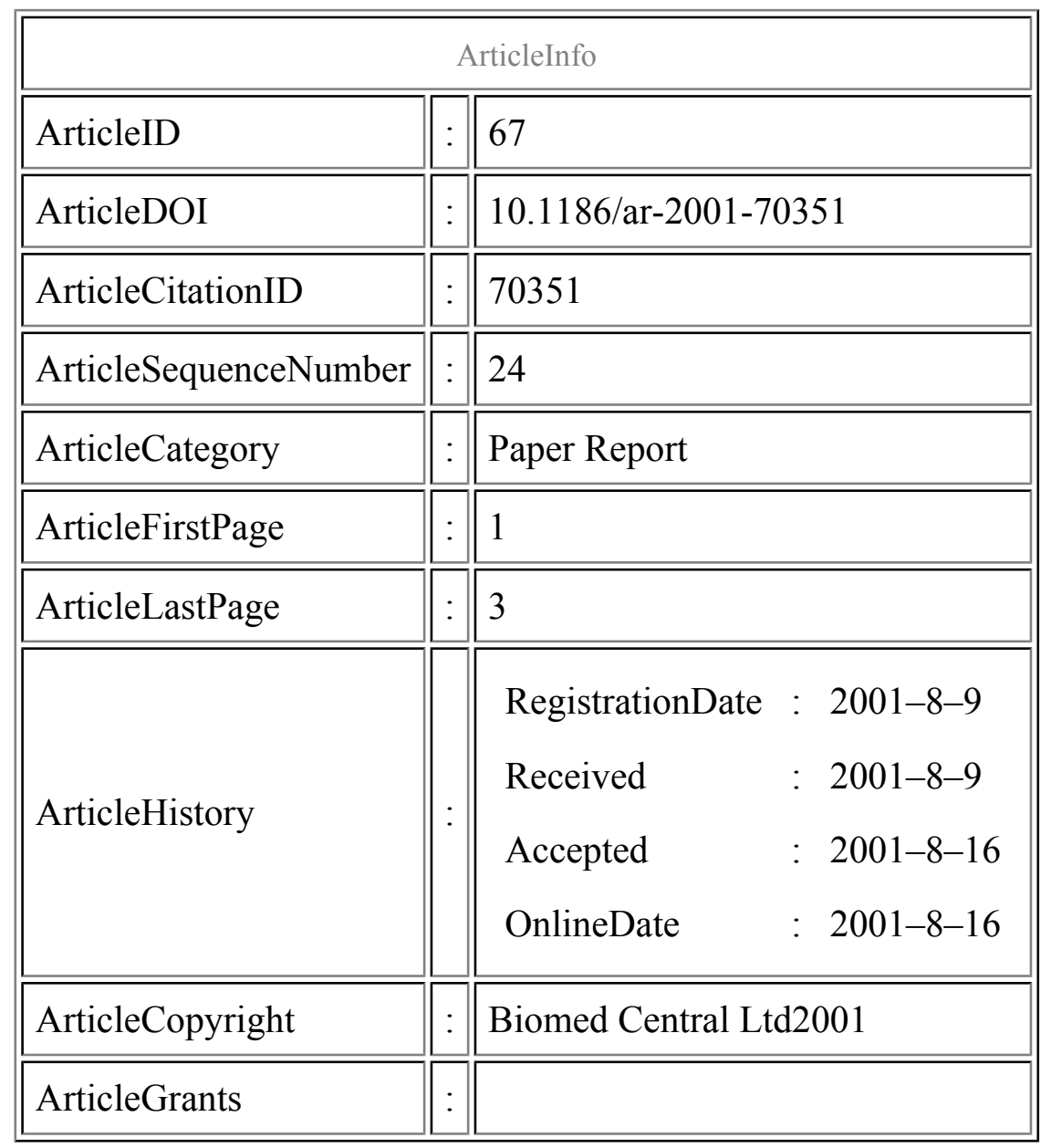




\begin{tabular}{|l|l|l|l|}
\hline ArticleContext & $:$ & 130753311 \\
\hline
\end{tabular}

Thomas Pap, ${ }^{\text {Aff1 }}$

Aff1 University Hospital Magdeburg, Germany

\section{Keywords}

Rheumatoid arthritis, CD44, apoptosis, Fas

\section{Context}

Apart from the increased expression of adhesion molecules and matrix-degrading enzymes, alterations in apoptosis are characteristic for rheumatoid arthritis synovial fibroblasts (RA-SFs). Recently, it has been shown that cross-linking of CD44 on RA-SFs triggers the upregulation of disease-relevant molecules such as vascular cell adhesion molecule (VCAM)-1. Also, inhibition of CD44 decreases the invasiveness of RA-SF into cartilage and reduces the severity of arthritis in animal models. The authors investigated the effects of CD44-mediated signalling on the expression of Fas, one major receptor involved in the induction of apoptosis. They also studied whether cross-linking of CD44 through specific antibodies or hyaluronan affects Fas-induced apoptosis in RA-SFs.

\section{Significant findings}

Cross-linking of CD44 on RA-SFs increased the transcription of Fas mRNA, as well as the surface expression of Fas. Maximum levels of Fas receptor were seen 3 hours after cross-linking of CD44, and Fas expression returned to the values for unstimulated cells after 24 hours. Stimulation with IL-1? or TNFa as well as cross-linking of MHC-I, intracellular adhesion molecule (ICAM)-1 or VCAM-1 had so significant effects on the expression of Fas. Cross-linking of CD44 on RA-SFs also increased the susceptibility of RA-SFs to apoptosis induced by monoclonal antibodies to Fas. Interestingly, stimulation with native hyaluronan did not result in a significant change in the amount of programmed cell death, whereas the fragmented 6.9-kDa form increased the rate of Fas-mediated apoptosis.

\section{Comments}


Although this paper does not necessarily demonstrate that "CD44 is the physiological trigger of Fas up-regulation ...", it provides a potential mechanism that helps to explain earlier findings showing an increased expression of Fas on RA-SFs. The authors propose that in the hyaluronan-rich environment of the rheumatoid synovium, CD44 upregulates Fas on RA-SFs and sensitises these cells to Fas-mediated apoptosis. It is also suggested that CD44 signalling is distinct from signalling pathways triggered by proinflammatory cytokines such as TNFa and Il-1?. These findings are of interest as they link the expression of CD44 to the apoptotic machinery in RA-SFs. However, the authors do not discuss recent observations indicating that the susceptibility to apoptosis is not increased in some RA-SFs despite their high expression of Fas (see Additional information). Therefore, further investigations will be needed to explain the resistance of RA-SFs to Fas-induced cell death in the context of CD44, proinflammatory cytokines and potential intrinsic factors.

\title{
Methods
}

Culture of synovial fibroblasts, in vitro stimulation, flow cytometry, northern blot

\section{Additional information}

\author{
Pap T, Muller-Ladner U, Gay RE, Gay S: Fibroblast biology. Role of synovial fibroblasts in the \\ pathogenesis of rheumatoid arthritis. Arthritis Res 2000, 2:361-367 (PubMed abstract).
}

\section{References}

1. Fujii K, Fujii Y, Hubscher S, Tanaka Y: CD44 is the physiological trigger of Fas up-regulation on rheumatoid synovial cells. J Immunol. 2001, 167: 1198-1203.

This PDF file was created after publication. 